\title{
Muséologies
}

Les cahiers d'études supérieures

muséologies

\section{Entretien avec Bernard Deloche}

\section{Anik Meunier et Marie-Blanche Fourcade}

Volume 6, numéro 2, 2013

La cybermuséologie

URI : https://id.erudit.org/iderudit/1018932ar

DOI : https://doi.org/10.7202/1018932ar

Aller au sommaire du numéro

Éditeur(s)

Association Québécoise de Promotion des Recherches Étudiantes en

Muséologie (AQPREM)

ISSN

1718-5181 (imprimé)

1929-7815 (numérique)

Découvrir la revue

Citer ce document

Meunier, A. \& Fourcade, M.-B. (2013). Entretien avec Bernard Deloche.

Muséologies, 6(2), 53-62. https://doi.org/10.7202/1018932ar

Tous droits réservés (C Association Québécoise de Promotion des Recherches Étudiantes en Muséologie (AQPREM), 2013
Ce document est protégé par la loi sur le droit d'auteur. L’utilisation des services d'Érudit (y compris la reproduction) est assujettie à sa politique d'utilisation que vous pouvez consulter en ligne.

https://apropos.erudit.org/fr/usagers/politique-dutilisation/ 
Dialogue un

\section{Entretien avec Bernard Deloche}

Anik Meunier et Marie-Blanche Fourcade 
Bernard Deloche est professeur émérite à l'Université Jean Moulin - Lyon 3 où il a notamment dirigé pendant dix ans le master 2 de muséologie. Il est membre du Conseil international des musées (ICOM). Philosophe et muséologue, il est l'auteur de plusieurs contributions incontournables: parmi les plus récentes: Mythologie $d u$ musée: de l'uchronie à l'utopie (2010), La nouvelle culture: la mutation des pratiques sociales ordinaires et l'avenir des institutions culturelles (2007), Le musée virtuel. Vers une éthique des nouvelles images (2001). 
Depuis les vingt dernières années, vous vous intéressez aux rapports entre nouveaux médias et musées. Comment, selon vous, caractériser la relation qu'ils entretiennent?

Bernard Deloche - Sans conteste, et aussi étonnant que cela puisse paraître car musées et médias sont placés sur deux terrains parfaitement hétérogènes que tout semble opposer, il s'agit pour moi d'une relation de concurrence, le musée étant souvent tourné vers les témoins du passé alors que les médias se trouvent au cœur de l'actualité la plus vivante. Mais, attention, la concurrence n'est pas là où on le croit généralement. On pense parfois que le musée en ligne ou le cybermusée est tout simplement en train de remplacer le musée institutionnel, avec tout le cortège de conséquences qui s'ensuit: baisse de la fréquentation et baisse des crédits qui peuvent aller, dans les cas extrêmes, jusqu'à entraîner la fermeture du musée lui-même, la grande frayeur des conservateurs. Or il n'est absolument pas question de cela, mais bien d'une concurrence entre différents médias : le média-musée - déjà considéré comme tel par Marshall McLuhan en 1967 - face aux médias technologiques que sont les moyens informatiques et leur outil majeur d'aujourd'hui, Internet, que l'on qualifie de nouveaux médias.

\section{Effectivement vous évoquez à plusieurs reprises dans vos} travaux la notion de concurrence entre nouveaux médias et musées. Avec l'usage massif des technologies et leur intégration exponentielle dans les musées, avez-vous constaté une redéfinition de cette concurrence dans la dernière décennie?

B.D. La question n'est pas celle d'un usage plus ou moins massif, mais plutôt celle de la nature de l'action exercée, car il est évident que celle-ci a profondément changé durant les quinze dernières années. Entendonsnous bien, ce n'est pas au sein du musée que s'exerce la concurrence la plus redoutable, mais à l'extérieur par l'action produite par les différents médias sur la culture. Le musée, depuis ses origines, n'a cessé de fonctionner comme un média et, comme tel, il a toujours généré des modèles culturels. Mais on ne doit pas oublier que, pendant des siècles, la civilisation occidentale a été dominée par le monopole de l'Église catholique qui contrôlait à la fois la distribution des connaissances et celle des images. Elle était, de fait et en droit, l'autorité culturelle officielle, c'est elle qui décidait de la réponse aux trois questions que posera Kant à la fin du XVIII siècle: que puis-je savoir? que dois-je faire? qu'ai-je le droit d'espérer? Elle façonnait ainsi les pratiques sociales. Or le musée est apparu officiellement avec la Révolution française, qui a substitué au monopole de l'Église le double monopole de l'École (en charge des connaissances) et du musée (en charge du visuel). Le musée est ainsi devenu l'une des deux grandes références officielles de la culture. Il suffit pour s'en convaincre de considérer les thèmes des collections de peintures des musées traditionnels : les héros et les dieux (païens ou chrétiens), les princes et les capitaines, les grands faits historiques, mais aussi la belle nature. Bref, les modèles proposés par le musée faisaient autorité en toutes circonstances et on allait au musée pour s'humaniser. 
De leur côté, les médias, qu'ils soient classiques - comme la presse, la radio ou la télévision - ou nouveaux - comme Internet, l'ordinateur et les supports électroniques de stockage, cédérom, DVD, etc. -, n'ont cessé de proposer, eux aussi, des modèles culturels. Mais ces derniers modèles sont sensiblement différents de ceux que véhiculait le musée, d'où un impact également différent sur la vie sociale. Ces nouveaux médias produisent et diffusent une nouvelle culture. J'ai bien dit produisent, car il ne s'agit pas simplement d'un processus d'élargissement ou d'accélération de la diffusion : c'est le contenu lui-même qui change, c'est-à-dire la culture au sens fort. Non pas les qualités individuelles de telle ou telle personne réputée cultivée (les connaissances, la sensibilité, le raffinement, etc.), mais le processus collectif qui inclut les modes de vie, les manières de penser et le système de valeurs d'un groupe social.

\section{Permettez-moi de faire ici un petit inventaire de ces nouveaux modèles:}

1 - Il s'agit d'abord de modèles identitaires. Avec les premiers médias de masse, la concurrence est apparue: la publicité et les magazines de mode ont substitué les vedettes du show biz et les dieux du stade à ceux de la religion et de la mythologie. Si les objets avaient changé, le processus demeurait formellement le même, au point qu'il était encore possible de concilier les anciens modèles avec les nouveaux (aller à la messe, visiter le musée, lire le magazine de mode ou le journal de sport). En revanche, il semble que tout ait basculé avec les médias technologiques d'aujourd'hui (d'abord la télévision, puis le cédérom et le Web, etc.), car ils ont introduit un nouveau type de modèles, en promouvant le spectateur lui-même au rang de modèle, selon un principe qui fonctionnait déjà implicitement dans les musées d'anthropologie, à savoir le gommage de toute transcendance. En effet, ces musées, plus récents que les musées d'art ou les musées scientifiques et techniques, avaient déjà mis la vie quotidienne en vitrine et, du même coup, changé les objets d'identification proposés au visiteur. Dans ces musées déjà je n'admirais plus quelque chose qui m'est étranger (les dieux, les saints ou les princes), mais je me contemplais moi-même, en vitrine, sous les traits d'un autre. Avec les nouveaux médias, c'est moi qui suis la vedette, comme le suggérait de façon emblématique ce jeu publicitaire diffusé par Nestlé en 2001, Imagine-toi en héros, qui proposait de placer sa propre photographie dans une fenêtre réservée à cet effet pour se voir déguisé en héros. Je ne rêve plus que je suis un autre, je me découvre moi-même dans ma particularité comme un être d'exception et, du rêve d'autrefois, je sombre dans le délire. On constatera que les médias d'aujourd'hui ne font rien d'autre: avec la webcam (la live cam notamment), je suis moi-même le héros d'un film que tout le monde peut voir sur le Net; de même, avec la téléréalité, c'est l'intimité de chacun, ses joies et ses peines, ses problèmes quotidiens les plus prosaïques, sa nudité même, qui sont exhibés aux yeux de tous. Toute dimension de normativité s'est effacée au profit d'une exorbitante valorisation de soi.

2 - Mais ils produisent aussi des modèles comportementaux. Pierre Bourdieu parlait de schème ou de modus operandi. Ce qui veut dire que ce qu'on nous montre, comme la manière dont on nous le montre, 
tendent à induire des pratiques sociales spécifiques. Or il y a un modus operandi généré par les médias: emblématique, le mode d'intervention et de circulation dans le réseau du Net (le principe de la relation hypertexte notamment) révèle une rupture radicale de schème dans la civilisation occidentale avec l'apparition des comportements interactifs et non linéaires. Linéaire, le trajet du rat qui explore un labyrinthe ou une arborescence, car il suit un parcours préétabli; non linéaire au contraire celui du singe (ou de Tarzan) qui saute de branche en branche. L'hypermédia à l'intérieur d'un site Web relève d'un parcours arborescent et linéaire, tandis que le mode d'exploration suggéré par Internet est absolument non linéaire et conforme à l'image du rhizome exploitée par Gilles Deleuze et Félix Guattari. Avec les médias technologiques, la non-linéarité est enfin reconnue, sinon assumée, pour elle-même. Non linéaire ne signifie pas déstructuré, mais structuré autrement ou, plus exactement, structuré dynamiquement. C'est la tactique opposée à la stratégie. Dans le contexte actuel de la mondialisation, elle s'est révélée autrement plus efficace. C'est ainsi que, de nos jours, il est de plus en plus rare qu'un jeune envisage de faire toute sa carrière professionnelle dans le même métier, voire passer toute sa vie avec le même conjoint. Telle est la culture induite par les médias et, là, il est évident que la concurrence est manifeste.

3 - Enfin ces médias produisent également des modèles mentaux, c'est-àdire de nouveaux processus de pensée consécutifs à la multiplication illimitée et déstructurée des images. Reste à savoir quel est l'impact de cette multiplicité d'images sur les pratiques sociales. Difficile et coûteuse, longue à produire, l'image du passé avait une valeur propre résultant du travail qu'elle avait demandé et on comprend qu'elle soit spontanément devenue un objet de culte: celle d'aujourd'hui est présente à tout instant sous nos yeux et nous ne pouvons pas risquer un pas dans la rue sans tomber sur une affiche publicitaire qui nous propose une image. Que l'homme de la Renaissance ait vu son mental structuré par ce qu'il percevait, rien d'étonnant à cela, car la rareté des images faisait de l'expérience perceptive une expérience forte, marquante. En ce sens le producteur des images, l'artiste, jouait un rôle démiurgique en façonnant le regard des spectateurs. Mais l'homme de la société industrielle avancée est-il encore marqué par ce qu'il perçoit? Il l'est plutôt par la discontinuité et le désordre dans lesquels se présentent les images. Ce qui explique peut-être que les enfants d'aujourd'hui aient plus de difficulté à concentrer leur attention ou peut-être même à conceptualiser, dans la mesure où le mode de conceptualisation qui leur est proposé par les images ne fonctionne plus par induction, abstraction et généralisation (remonter des exemples à l'essence), mais, comme le définit Gilles Deleuze, par agrégat d'éléments hétérogènes. C'est donc une nouvelle démarche intellectuelle qui se dessine et qui se trouve manifestement en conflit, voire en contradiction, avec les méthodes d'enseignement linéaire de l'école. Ce désordre, que l'on sera tenté de dénoncer, est en réalité fort exigeant, car il renvoie à des modes d'appropriation à la fois métastables et multivariés, dans lesquels il revient à chacun de se construire ses propres axes et pôles de référence. 
La culture vivante actuelle est ainsi produite et diffusée par les médias, alors que le musée continue à répandre la culture d'autrefois, devenue objet de curiosité, au même titre que la peinture italienne de la Renaissance pour un touriste Japonais. Désormais, le muséographe et le commissaire d'expositions sont directement concurrencés par le publicitaire, l'animateur de télévision et même par le webmestre, devenus les maîtres de la distribution des images.

\section{Selon vous, l'entrée des nouvelles technologies, sous diverses formes et différentes modalités, contribue-t-elle à transformer l'identité du musée?}

B.D. Oui et non, car ce n'est pas leur entrée au musée qui transforme directement le musée. Même si ces nouveaux outils envahissent de plus en plus les salles d'expositions, le phénomène est très secondaire. Ainsi, pendant des années, le microordinateur dans bien des musées n'était utilisé que comme un substitut assez efficace de la machine à écrire, aussi restait-il confiné dans les secrétariats. Par exemple, nul n'imaginait ou ne voulait imaginer que l'ordinateur pouvait devenir un outil de connaissance des collections, car celles-ci étaient réputées sacrées, c'est-à-dire inaccessibles par essence à une démarche cognitive et donc scientifique. Selon moi, le Musée national des arts et traditions populaires à Paris a été l'un des tout premiers musées à informatiser ses collections, mais cela ne fut possible que parce qu'elles étaient considérées comme étant à la limite de l'art et de l'utilitaire.

Le recours de plus en plus fréquent aux bornes interactives dans les musées depuis une quinzaine d'années me semble relever seulement des gadgets apparentés aux jeux vidéo, propres à attirer et à mobiliser l'attention des enfants et parfois même des plus grands. Ces instruments créent une sorte de motivation impure qui a pour effet de captiver un certain public par des moyens en définitive étrangers au contenu que le musée souhaite communiquer.

Ce n'est pas l'irruption des nouvelles technologies qui provoque par elle-même une mutation de l'identité du musée, mais bien le problème qu'elles posent et les réactions qu'elles provoquent. Car, lorsqu'il prend conscience de la concurrence que j'évoquais il y a un instant, ce qui est encore assez rare, le musée tend à réagir afin de reprendre sa place au sein du monde restreint des décideurs, de ceux qui produisent la culture, qui disent ce qu'elle doit être.

C'est un aspect, par exemple, des récents musées de société, qui ont pratiquement éliminé les collections d'objets sacrés pour recourir à des objets usuels afin de les faire "parler " et de provoquer ainsi la conscience du visiteur. Je pense en particulier à l'expérience d'un pionnier en la matière, Jacques Hainard, au Musée d'ethnographie de Neuchâtel (Suisse). Avec lui et avec ses successeurs, on a assisté à la disparition des expositions dogmatiques, c'est-à-dire destinées à faire passer un message au public, et cela au profit d'interrogations qui parfois restent sans réponse. Dans ces conditions, le musée agit sur 
la vie sociale en responsabilisant les visiteurs, d'où le choix de thèmes éthiques comme l'euthanasie, les organismes génétiquement modifiés (OGM), la gestion de l'eau, etc.

\section{Alors qu'elles sont désormais bien ancrées dans un grand nombre d'activités du musée et dans les différents secteurs de la société, les nouvelles technologies représentent-elles encore un défi de taille pour le musée?}

B.D. Cet ancrage dans les activités du musée peut-il être considéré comme un défi? Certes oui, car les nouvelles technologies parviennent à introduire la concurrence non plus seulement hors du musée en produisant de nouveaux modèles, mais bien en son sein même, ne serait-ce qu'en détournant l'attention du public qui, comme je le disais il y a un instant, se trouve plus intéressé par l'aspect technico-ludique de ces outils que par le message que l'on souhaite lui faire passer. Mais ce point me semble secondaire et superficiel par rapport à celui que je viens d'évoquer: on est là en face d'une concurrence habituellement reconnue et non en face de la vraie concurrence, celle des nouveaux décideurs de la culture et des pratiques sociales qu'ils génèrent.

Est-ce que les conditions d'accès que permettent les nouveaux médias, dans une logique de démocratisation de la culture, favorisent à votre avis le développement de nouvelles catégories de publics?

B.D. Il semble, en tout cas, que le développement des sites Internet de musées ne réduise pas, comme on le craignait au départ, la fréquentation physique des établissements. Il se pourrait même que cela contribue à la renforcer. Le visiteur peut choisir d'avance les priorités de sa visite. En outre, la mise en ligne des collections permet d'ouvrir au public toutes les réserves, ou seulement une partie de celles-ci..., car les musées qui exposent leurs réserves sont très rares et, souvent, on se sent frustré à l'idée que toutes ces richesses dorment au fond des caves. De cette manière, grâce à la complémentarité des deux types de visites, on arrive à montrer "tout à tous ", conformément au rêve démocratique du comte de Clarac, conservateur du département des sculptures du Louvre au milieu du XIX ${ }^{\mathrm{e}}$ siècle. Cela signifie-t-il que le champ des publics est en train de s'élargir et de se diversifier? Rien n'est sûr en ce domaine, comme l'ont montré les travaux déjà anciens de Pierre Bourdieu. Par exemple, les nouveaux publics sont plutôt attirés par l'art contemporain avec lequel ils se sentent davantage en consonance. Mais je ne crois pas que ce soit là l'effet des nouveaux médias, car le germe de l'art contemporain est apparu à l'aube du $\mathrm{XX}^{\mathrm{e}}$ siècle avec des personnalités tel Marcel Duchamp: il est donc beaucoup plus ancien.

On dit qu'Internet a considérablement transformé les formes du lien social. Dans le cas du musée, cette affirmation est-elle aussi valable? Les nouvelles technologies, qu'elles soient utilisées au sein des musées ou qu'elles soient les supports des formes muséales (par exemple les musées en ligne), ont-elles modifié la relation des publics aux institutions, voire des publics entre eux? 
B.D. Il est manifeste, en effet, qu'Internet agit sur les pratiques sociales comme sur les mentalités, non seulement en facilitant l'accès aux connaissances, mais surtout en permettant à chacun de s'exprimer et d'être entendu. Bref, Internet a enfin généralisé l'interactivité de la communication. Par exemple, l'impact de ce mode d'expression sur la vie politique est manifeste et désormais les internautes parviennent à se grouper, à organiser des manifestations et même, en formant des contre-pouvoirs, à renverser des régimes institutionnels.

Dans les musées, au moins en théorie, la relation s'est considérablement transformée puisque la transmission (à sens unique du musée au public) tend à céder la place à une communication réciproque. Mais il est vrai que peu nombreux sont les musées qui la pratiquent, le seul retour du public s'exerçant le plus souvent par le biais du livre d'or devenu parfois un recueil de protestations. Ce sont sans doute d'abord les écomusées, dont on connaît le déclin spectaculaire, qui ont introduit l'interactivité. Mais, dans les années 1970, celle-ci ne pouvait pas résulter du développement d'Internet qui n'a été vraiment effectif que dans les années 1990. La forme de musée à la fois la plus interactive et la plus contemporaine d'Internet est représentée de nos jours par les musées de société qui, au moins, cherchent à substituer les questions aux affirmations. Une nouvelle forme de relation entre public et institution est donc encore à inventer.

Quant à la question de savoir si Internet a modifié la relation des publics entre eux, elle est très difficile à apprécier dans la mesure où l'on n'a actuellement qu'une connaissance très imparfaite du fonctionnement effectif des réseaux sociaux qui, paradoxalement, isolent les internautes tout en les rapprochant.

\section{Selon vous, l'inscription des médias au sein des musées appelle- t-elle de nouvelles formes de médiation?}

B.D. Non, je ne vois pas de nouvelles formes de médiation autres que la nécessité pour les musées de convertir leur propre médiation pour reprendre la main face à une diffusion incontrôlée de nouveaux modèles. Ils tentent de le faire aujourd'hui par trois moyens : l'ouverture à la diversité et au dialogue entre les cultures (métissage), le renoncement au culte des collections au profit d'une improvisation des parcours (non-linéarité) et le privilège accordé à l'interrogation plutôt qu'à l'acculturation (interactivité).

Après près de vingt ans de réflexion sur la question, est-ce que vous estimez que ces nouvelles formes de médiation ont atteint une certaine maturité, sinon une légitimité au sein des musées?

B.D. À en juger par ce que j'ai pu observer autour de moi, ces nouvelles formes de médiation sont encore rares et ont grand peine à s'imposer dans le paysage culturel, comme en témoigne par exemple l'échec de l'entreprise qu'avait tenté d'initier Michel Colardelle à Marseille (le Musée des civilisations de l'Europe et de la Méditerranée, MuCEM). 


\section{À votre avis, comment le musée a-t-il reçu et reçoit-il encore cette vague de nouvelles technologies? Avez-vous identifié des pièges avec lesquels le musée est aux prises? Sinon, des "potentialités" sur lesquelles le musée tente de capitaliser?} B.D. Manifestement le musée a considérablement évolué face aux nouvelles technologies de l'information et de la communication: après les avoir fuies comme dangereuses et même subversives, il a compris l'avantage qu'il pouvait en tirer et il a commencé à les accepter en tentant parfois de les récupérer à son compte, à mon avis sans grand succès. Il faut savoir qu'une lourde inertie pèse sur les musées: inertie administrative souvent liée à l'enracinement dans le service public, mais aussi inertie morale et psychologique, qui rendent extrêmement difficile une exploitation optimale des médias technologiques. C'est ainsi que les sites Web des musées oscillent habituellement entre le culte sacralisant, le purement didactique et le purement ludique. Ils oublient que leur grande spécificité est le caractère visuel irremplaçable de la culture qu'ils véhiculent. Leur difficulté est sans cesse de se distinguer des autres visuels fournis par les nouveaux médias, car tous ont un impact considérable, souvent beaucoup plus efficace que celui des musées eux-mêmes, notamment en raison de leur accessibilité simplifiée.

\section{Pensez-vous qu'il existe aujourd'hui ce que certains appellent la "cybermuséologie»?}

B.D. Vous me permettrez d'abord de vous féliciter de parler de cybermuséologie et de cybermusées plutôt que de "musées virtuels " comme on l'entend trop souvent. Car le virtuel ne s'oppose pas au réel, mais à l'actuel: de plus il n'a rien à voir avec les machines à information ou avec les nouvelles techniques, il n'appartient pas aux progrès de la science et de la technique, mais plutôt à l'archéologie muséale, c'est-à-dire aux diverses expériences de mise entre parenthèses de telle ou telle dimension du musée institutionnel: le "musée imaginaire " d'André Malraux est une figure du musée virtuel, mais il n'a rien à voir avec le cybermusée.

Il y a effectivement aujourd'hui des travaux consacrés au cybermusée, parfois appelé à tort musée virtuel. Mais s'agit-il vraiment de traités de muséologie spécifiques? Non. Généralement, ils se contentent de décrire de façon plus ou moins admirative les nouvelles techniques: par exemple, la cybervisite sur CD d'une exposition au Louvre dans laquelle on a poussé le raffinement jusqu'à vous faire entendre le parquet de bois qui grince sous les pieds du visiteur... Rien de cela ne relève de la muséologie ni même de la muséographie, qui gère les modalités techniques de la présentation. La muséologie traite des enjeux philosophiques, esthétiques, éthiques et même politiques, non des modalités d'utilisation de tel ou tel média. Tant que ces enjeux ne sont pas pris en considération, il n'est pas possible de parler de muséologie. À mon sens, il n'y a pas de cybermuséologie à proprement parler, mais la muséologie évolue dans sa conception du musée en fonction du nouveau contexte créé par les médias.

Dans le cadre de vos responsabilités liées au master de muséologie et nouveaux médias, quel bilan tirez-vous de la formation des 
jeunes muséologues? Sont-ils préparés à faire face et à accompagner cette dynamique muséologie/nouvelles technologies?

B.D. J'ai effectivement fondé en 1999 et dirigé pendant dix ans, à l'Université Lyon 3, un master 2 intitulé "Muséologie et nouveaux médias". L'expérience de cette formation a été double: elle m'a révélé, d'une part, l'excellente réceptivité des étudiants aux idées nouvelles (je pense à leur enthousiasme lors d'une visite de Jacques Hainard) et, d'autre part, la difficulté qu'ils ont rencontrée lors de leur stage dans les musées. En France au moins, les conservateurs de musées étaient et sont encore extrêmement peu préparés à sortir des sentiers battus: de plus, ils sont souvent entravés par leur autorité de tutelle, qu'il s'agisse de la municipalité, du département, de la région ou même de l'État. Les étudiants, pleins de dynamisme et d'espoir, ont été souvent déçus par la réalité institutionnelle devant laquelle ils se trouvaient tout d'un coup. Dans le meilleur des cas, on les a chargés de travailler sur des problèmes d'ordre muséographique, comme la mise en place de bornes interactives ou l'utilisation du téléphone portable comme audioguide. Les responsables de musées français ne sont pas encore très réceptifs à l'idée d'une vraie réflexion sur les enjeux.

\section{En tant que philosophe et muséologue, quel regard portez-vous} sur le musée actuel et celui de demain?

B.D. Face à cette mutation sociale qui s'ébauche sous nos yeux et à cette étonnante concurrence, on peut se demander où va le musée institutionnel. Va-t-il disparaître ou est-il en train de connaître une nouvelle mutation? La réponse à ces questions est loin d'être simple et univoque: d'une part, il semble que le triplet "métissage / non-linéarité / interactivité " ait inspiré et continue d'inspirer les musées en suggérant des pratiques muséales calquées sur le nouveau modèle des pratiques sociales, bref des musées qui acceptent l'échange et l'imprévu. Et, face aux phénomènes de société qui avaient déplacé les sources de la culture, le musée a essayé de rebondir en s'inventant une fonction critique. Renonçant à son rôle de producteur et distributeur de la culture, il tend à se muer en observateur vivant des faits sociaux, non comme un miroir identitaire et narcissique, mais comme un miroir interactif, c'est-à-dire un facteur de prise de conscience. Plus modeste que naguère, le musée ne prétend plus générer ou définir les pratiques sociales elles-mêmes, il veut seulement aider à les comprendre. Ainsi, non seulement les médias technologiques ont bousculé le champ culturel, mais ils ont également engendré une mutation du musée proprement dit pour en faire une sorte de laboratoire des faits sociaux.

\section{Aimeriez-vous ajouter quelque chose ou compléter certains des éléments qui ont été soulevés lors de cette discussion?}

B.D. Non, je n'ai rien à ajouter, sinon un vœu, celui de voir une accélération du processus d'évolution des musées vers le modèle des musées de société. Certains signes, parfois timides, le laissent espérer : par exemple, de plus en plus de musées de beaux-arts, renonçant aux "grand messes " de jadis (Raphaël à Paris, au Grand Palais, ou Vermeer à La Haye, etc.), organisent des expositions thématiques dans lesquelles les collections sont mises au service d'une interrogation réellement en prise sur la vie. 